\title{
Vagus Nerve Stimulation Suppresses Generalized Seizure Activity and Seizure-Triggered Postictal Cardiac Rhythm Changes in Rats
}

\author{
D. SAHIN ${ }^{1}$, G. ILBAY ${ }^{1}$, M. IMAL ${ }^{1}$, O. BOZDOGAN ${ }^{2}$, N. ATES ${ }^{1}$ \\ ${ }^{1}$ Kocaeli University, Faculty of Medicine, Department of Physiology, Kocaeli, ${ }^{2}$ Abant Izzet Baysal \\ University, Faculty of Science, Department of Biology, Bolu, Turkey
}

Received July 5, 2007

Accepted June 30, 2008

On-line July 25, 2008

\begin{abstract}
Summary
In the present study, we investigated the effects of vagus nerve stimulation (VNS), a proposed treatment for patients with intractable epilepsy, on cardiac rhythm following seizures induced by pentylenetetrazole (PTZ) in Wistar rats. After a baseline recording of electroencephalogram (EEG), electrocardiogram (ECG) and blood pressure (BP), rats in the first group received a single convulsive dose of PTZ (70 mg/kg) (Group 1). In the other two groups, the Wistar rats were implanted with a cuff electrode on the left cervical vagus nerve. One day after surgery, rats in the second group were treated with VNS (Group 2), whereas rats in the third group were connected to the stimulator but did not receive VNS (Group 3). Ten minutes after VNS onset, $70 \mathrm{mg} / \mathrm{kg}$ dose of PTZ was injected. EEG, ECG and BP were continuously recorded during post-injection period. Seizure severity was scored behaviorally. Then, baseline, ictal and postictal periods were analyzed for cardiac rhythms, seizure severity and blood pressure variability. PTZ treatment induced tonic-clonic seizure activity in all animals of Group 1 and Group 3. In these groups a marked increase of mean arterial blood pressure (MABP) but a significant decrease in heart rate and PP interval fluctuations was observed at postictal period. However, in the VNS-treated group the seizure scores and cardiac parameter returned to the baseline level. Present results emphasize that VNS effectively reduces seizure severity and suppress the seizure-induced cardiac rhythm changes.
\end{abstract}

\section{Key words \\ ECG • EEG • Epilepsy • Rat • Vagus nerve stimulation}

\section{Corresponding author}

N. Ates, Kocaeli University, Faculty of Medicine, Department of Physiology, Umut Tepe, Kocaeli,-Turkey. Fax: +90 2623037003. E-mail: sahindeniztr@yahoo.com

\section{Introduction}

Epilepsy is the second most common chronic neurological disorder after stroke affecting approximately $0.5-2 \%$ of the population. Seventy percent of patients can be successfully treated with one or more antiepileptic drugs. Despite adequate antiepileptic treatment $30 \%$ of patients continue to have seizures or experience unacceptable pharmacological side effects. For these patients with 'medically refractory' epilepsy, epilepsy surgery is a therapeutic alternative. Unsuitable candidates for resective surgery have few options left. Administration of a new antiepileptic drug will lead to seizure freedom in a maximum of $7 \%$ of patients (Boon et al. 2001, Dooley and Plosker 2000).

Electrical stimulation of the vagus nerve (VNS) is an efficacious neurophysiological treatment for patients with refractory epilepsy who are unsuitable candidates for curative resective surgery or who have experienced insufficient benefit from such a treatment (Boon et al. 2001, Salinsky et al. 1996, De Herdt et al. 2007). Several studies showed that VNS reduces generalized single or recurrent seizures both in human and experimental conditions (Krahl et al. 2003, Kostov et al. 2007, Zabara 1992). Some patients with epilepsy have now had a chronic implantation around the left vagus nerve of bipolar stimulating electrodes attached to an implantable stimulator resembling a cardiac pacemaker for the purpose of chronic and intermittent vagal stimulation (Wheless and Maggio 2002, Boon et al. 2001).

Naritoku et al. (1995) showed that VNS induces severe c-fos expression in brain stem and limbic structures such as amygdala and hippocampus which are 
highly epileptogenic and play an important role in the generalization of seizures.

The intricate relationship of the vagus nerve to cardiac function raises concern that vagal stimulation may affect cardiac rhythm and function (Krahl et al. 2001). Seizures are associated with marked autonomic changes during ictal, interictal and postictal periods which may be partly responsible for the production of cardiac arrhythmias and sudden unexpected death in epilepsy (Zijlmans et al. 2002, Wannamaker 1985, Wasterlain 1974).

Generalized convulsive seizures are associated with severe increases in blood pressure and conduction velocity of heart (Ilbay et al. 2003, Schraeder and Lathers 1989). The causes of death in epilepsy are commonly suspected to involve cardiovascular or respiratory dysfunction provoked by seizures (Sakamoto et al. 2008). Some abnormalities in neural regulation of cardiac rhythm during the immediate postictal state have also been described in animal experiments (Darbin et al. 2002).

Although the systemic effects of VNS application during the ictal and postictal state of generalized seizures have been studied extensively, specific approach to the cardiac arrhythmia at different seizure stages need to be further investigated. This study aimed to evaluate the relationship between VNS and postictal cardiac rhythm. Therefore we studied the acute convulsive seizures induced by pentylenetetrazole (Velíšek 2006) in rats to assess the influences of left VNS on the cardiac rhythm following this approach.

\section{Methods}

\section{Preparation of animals}

Male Wistar rats weighing 250-300 g were used as experimental subjects. Animals were housed in groups with free access to water and standard rat food and kept under a 12-h light-dark cycle. All experimental procedures were carried out with the approval of the Kocaeli University Ethics Committee.

Three electrodes (Plastic Products Company, MS 333/2A) were implanted under complete ketamine (100 mg/kg, i.p.) / chlorpromazine (1 $\mathrm{mg} / \mathrm{kg}$, i.p.) anesthesia. The coordinates to bregma were: A 2.0, L 3.5; A -6.0, L 4.0 in compliance with rat brain atlas (Paxinos and Watson 1998). The reference electrode was placed on the cerebellum. Then the electrodes were fixed by placing two screws in the skull using cold acrylic. To protect the electrodes from clogging, they were covered with dust cups until they were used.

\section{Experiments}

One week after the surgical procedure, rats were divided into three groups each containing 7 rats. In group 1 (PTZ administration), a PE-10 catheter fused to PE-50 catheter (Clay-Adams, Sparks, MD, USA) was inserted into the femoral artery under diethyl ether anesthesia, the other tip of the catheter was passed subcutaneously through the back of the animal and fixed at the level of the neck for monitoring blood pressure (BP). Subcutaneous needle electrocardiogram (ECG) electrodes were placed under the skin to record standard electrocardiogram. After a recovery period, EEG, ECG and BP were monitored with a data acquisition system (DA 100B; Biopac Systems) and 30 min later the animals were administered $70 \mathrm{mg} / \mathrm{kg}$ i.p. PTZ to induce generalized tonic-clonic seizures. Electroencephalogram (EEG), bipolar ECG and BP were continuously recorded during epileptic activity for 1 hour and stored in computer for future analysis. In group 2 (VNS condition + PTZ administration), one day before the experiments, rats were anesthetized with ketamine/xylazine $(75 / 15 \mathrm{mg} / \mathrm{kg})$, and an incision was made on the midline of the ventral neck. The cervical vagus nerve was isolated on the left and cuff electrode was implanted, wires from the cuff electrode were tunnelled under the skin and fixed at the level of the neck. One day after surgery, rats were connected to a stimulator and VNS (1-mA, 20-Hz, 500- $\mu \mathrm{s}$ square-wave pulses) was started in half of the rats with cuff electrodes. After $10 \mathrm{~min}, \mathrm{PTZ}$ (70 mg/kg, i.p.) was injected, VNS remained on during the entire procedure. During the whole procedure, EEC, ECG and BP recordings were made in the same way as in Group 1. In group 3, rats (No-VNS condition + PTZ administration) did not receive VNS, but were subjected to sham operation and connected to the stimulator. Ten minutes later, PTZ (70 mg/kg, i.p.) was injected and the entire procedure was repeated as described.

The effects of PTZ were quantified from the EEG recordings. The rats showed generalized tonic and clonic convulsions with continuous high-voltage spikes and sharp wave complexes on the cortical EEG. Behavioral convulsive activity confirming EEG seizure activity was observed and scored simultaneously. For the seizure scoring, the scale described by Velíšek et al. (1992) was used: stage $0=$ no change in behavior; stage 0.5 = atypical behavior (e.g. intensive grooming, sniffing, 


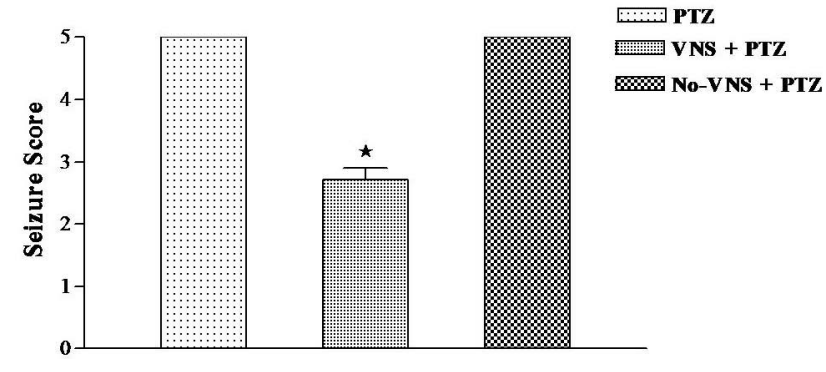

Fig 1. Effects of VNS on PTZ-induced seizure severity vs. PTZ application and No-VNS+PTZ application $* p<0.05$. Note that VNS induced a significant decrement in the seizure score.

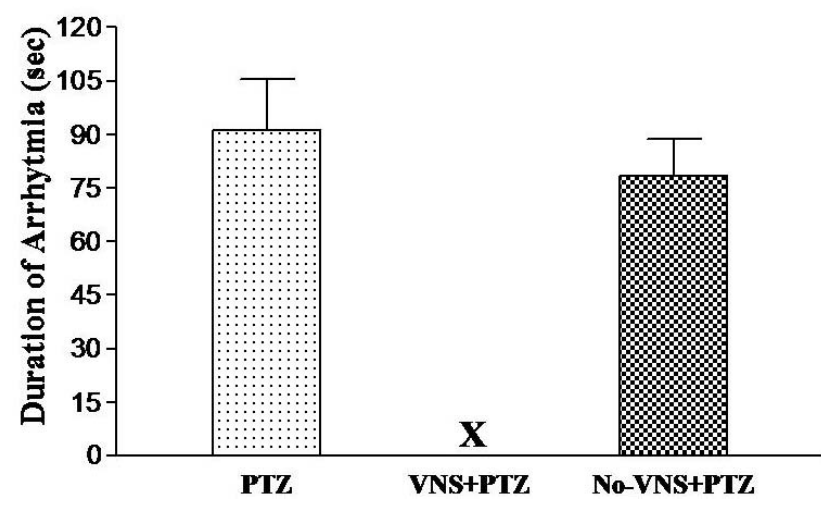

Fig 2. Inhibiting effect of VNS on PTZ-induced cardiac arrhythmia duration.

moving arrests); stage $1=$ isolated myoclonic jerks and ear and facial twitching; stage 2 = atypical minimal seizures and convulsive waves throughout the body; stage 3 = fully developed minimal seizures, clonus of the head muscles and forelimbs, and the presence of the righting reflex; stage 4 = major seizures (i.e. generalized, without the tonic phase); and stage $5=$ generalized tonic-clonic seizures beginning with wild running, followed by lost righting ability and tonic phase (i.e. flexion or extension of forelimbs and hindlimbs) progressing to the clonus.

Offline, the ECG and BP recordings were analyzed using the Biopac acquisition and analysis system. Arterial pressures reported are mean arterial blood pressures $(\mathrm{MABP})$, where $\mathrm{MABP}=$ (diastolic pressure $+(1 / 3$ systolic pressure - diastolic pressure $))$. Because of motion artifact during seizure activity, it was not possible to obtain a reliable time for arrhythmia onset, but heart rate and PP interval changes were common. Thus, the seizure onset provided a reproducible reference point of arrhythmia onset. Arrhythmia was apparent on ECG during the ictal and postictal period. The return to a regular rhythm is easily observed on the ECG tracing. Subsequently, we defined the arrhythmia duration from the onset of the seizure till the return to a regular rhythm visualized on the ECG.

\section{Statistics}

The results were indicated as the mean \pm SEM. For the comparison of the PTZ, VNS condition + PTZ and no-VNS condition + PTZ groups, ANOVA followed by Dunn's test was used. As a basis for all statistical decisions, $P<0.05$ was considered statistically significant.

\section{Results}

PTZ treatment $(70 \mathrm{mg} / \mathrm{kg})$ induced generalized tonic-clonic seizures that started with the clonus of the facial and forelimb muscles (minimal clonic seizures), and continued with the neck and tail extensions, loss of righting reflex with tonic flexion-extension, wild running and usually with extended clonic activities. During the generalized tonic-clonic seizure, continuous high voltage spikes and sharp wave complexes were recorded on the cortical EEG. At the end of tonic-clonic seizures, the postictal depression was also evident on the EEG. The average seizure score and duration of arrhythmia in these groups are presented in Figures 1 and 2. Concerning the seizure severity and cardiac rhythm changes, no significant differences between these two groups were determined. The average seizure score was the same (stage 5), and duration of arrhythmia and postictal depression were similar in all animals $(91 \pm 14.5 \mathrm{~s}$, $78.5 \pm 10 \mathrm{~s}, 51 \pm 17 \mathrm{~s}, 47 \pm 15 \mathrm{~s}, \mathrm{PTZ}$ and no-VNS+PTZ groups, respectively). Blood pressure increased in rats receiving PTZ (baseline $106 \pm 2 \mathrm{mmHg}$; ictal $146 \pm 8$ $\mathrm{mm} \mathrm{Hg}$ ) and no-VNS+PTZ (baseline $109 \pm 3 \mathrm{~mm} \mathrm{Hg}$; ictal $145 \pm 10 \mathrm{~mm} \mathrm{Hg})$ during seizures $(\mathrm{p}<0.05)$, whereas BP fluctuation often occurred around postictal period (139 $\pm 20 \mathrm{~mm} \mathrm{Hg}$ and $137 \pm 17 \mathrm{~mm} \mathrm{Hg}$, respectively). There were no differences in heart rate during preinjection periods in all groups. However there was significant decrement in the heart rate during the postictal periods compared to the baseline in PTZ and no-VNS+PTZ groups ( $339 \pm 10$ vs. $184 \pm 23 ; 342 \pm 18$ vs. $209 \pm 20$ beats per minute, respectively, $\mathrm{p}<0.001)$. In these groups $\mathrm{P}, \mathrm{QRS}$ and $\mathrm{T}$ deflections were all normal, but there was some prolongation of the PP interval during the postictal periods. During the recovery of postictal depression, return to regular rhythm was observed on the ECG. Typical EEG, BP and ECG traces at the end of the seizures are shown in Figure 3A.

The application of VNS started $30 \mathrm{~min}$ before 
Fig 3.A

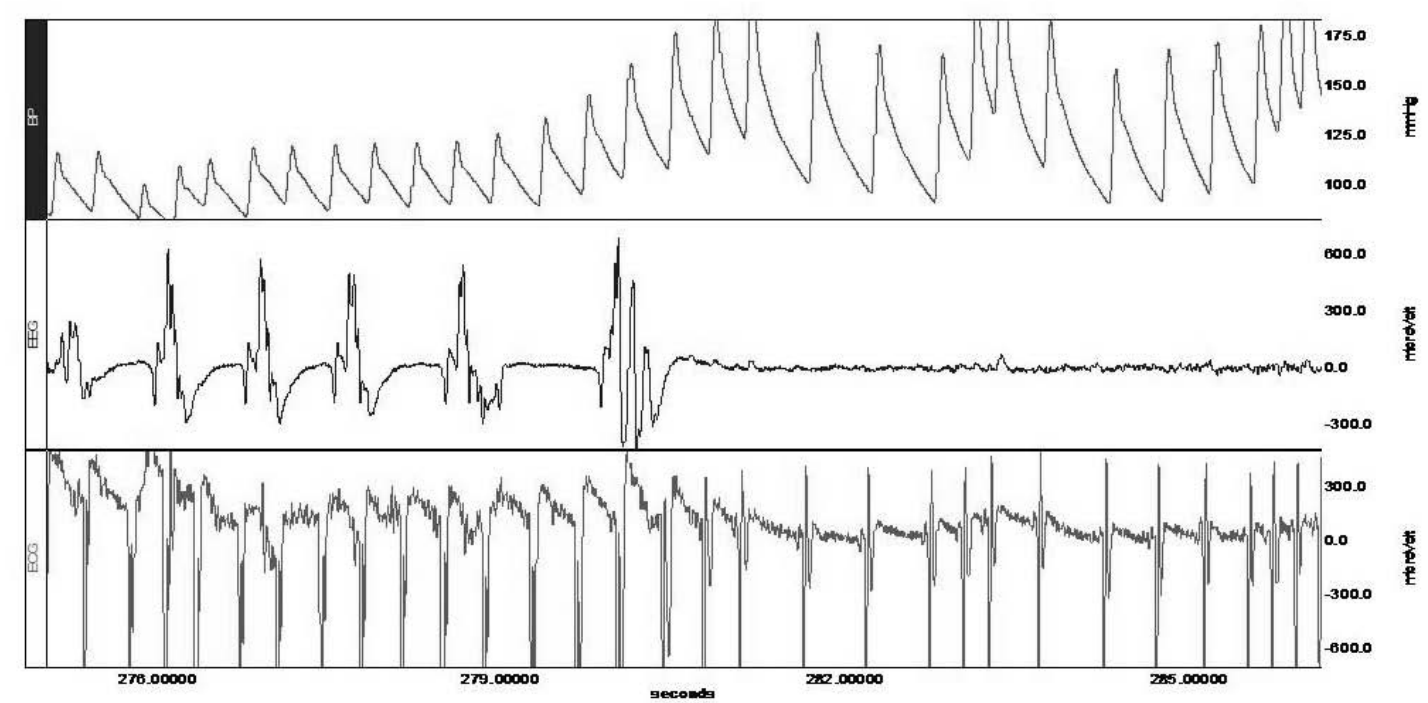

Fig 3.B

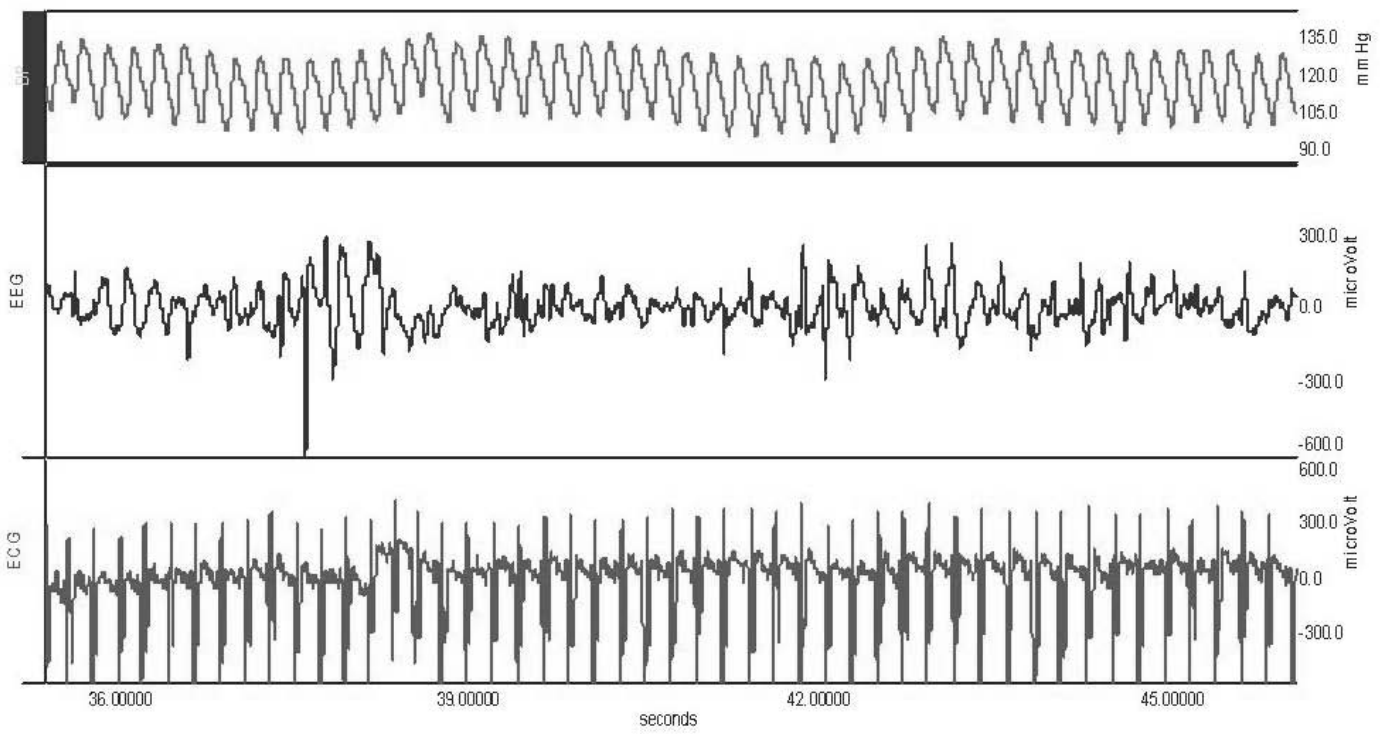

Fig 3. Sample recordings of cortical EEG, ECG and BP simultaneously. A. Blood pressure increment and arrhythmia with bradycardia during postictal depression period after PTZ-induced seizure without VNS. B. VNS prior to PTZ administration suppressed seizure severity but did not stop it completely. However, blood pressure and heart rate remained near baseline values.

PTZ treatment did not induce any cardiac abnormality. VNS prior to PTZ (Group 2) significantly decreased to the seizure severity and suppressed the expression of generalized component of convulsion that include wild running, and loss of righting ability. There were intermittent spike activities on the EEG records, which were behaviorally accompanied by minimal clonic seizures (Fig. 3B). In this seizure pattern, seizures started with atypical behaviour or isolated myoclonic jerks and facial twitching, and continued with fully developed minimal clonic seizures, clonus of the head muscles and forelimbs. Blood pressure and heart rate did not change significantly in rats under the left VNS condition (baseline $110 \pm 4 \mathrm{~mm} \mathrm{Hg}$, postictal $127 \pm 3 \mathrm{~mm} \mathrm{Hg}$; baseline $334 \pm 18$, postictal $382 \pm 21$ beats per minute) (Fig. 3B). No alterations in the PP interval and cardiac arrhythmia were detected.

\section{Discussion}

The results of this study showed that the VNS suppress to the seizure-related cardiovascular changes in PTZ-induced generalized tonic-clonic seizure. Generalized seizures are associated with autonomic 
neuronal dysfunction that result in abnormalities of heart rate and blood pressure (Darbin et al. 2002, Sahin et al. 2003) The autonomic changes could be influenced by the activation of some cortical structures involved in seizure activity and those structures of the central autonomic network, such as amygdala and hypothalamus. It was hypothesized earlier that cortical desynchronization induced by activation of unmyelinated C-fibres through the reticular activating system played a key role in the mechanism of action of VNS. Moreover, Vonck et al. (2001) showed that the activation of NST and locus coeruleus through electrical stimulation of the vagus nerve may cause release of seizure-suppressing neurotransmitters. Thus, the protective effects of VNS against cardiac arrhythmia may also be related with its antiseizure potency (Krahl et al. 2003, Boon et al. 2001).

The EEG suppression, simultaneous fluctuation of BP and cardiac bradyarrhythmia during the postictal periods were identified and documented in this study. Our observations show that removal of higher autonomic system activity is apparently influencing the cardiac function during the postictal period. This suggests that VNS treatment may assist the continuation of physiological stability in cardiac events which are associated with seizure activity. Alternatively massive sympathetic outflow that occurs in parallel with the vagal outflow may be somehow protective against seizureinduced cardiovascular changes (Sakamoto et al. 2008).

VNS afferents reach many different brain regions, such as nucleus tractus solitarii which regulates cardiac rhythm and blood pressure (Koenig et al. 2007). Considering the simultaneous fluctuation of blood pressure and cardiac abnormality, it is possible that activation of the afferent pathway during seizure has a wide-ranging effect on multiple systems and pathways. These findings are consistent with previous hypothesis showing that an increase in seizure severity results in the provocation of autonomic responses (Darbin et al. 2003).
There is a relatively high incidence of cardiac rhythm changes in the epileptic population. This relationship may partially account for the occurrence of SUDEP (Sudden unexpected death in epilepsy) seen in epileptic patients and suggests that anticonvulsants may have protective effects against seizure-evoked hemodynamic dysfunction by reducing seizure severity. The exact causes of SUDEP are unknown, but it has been hypothesized that SUDEP could be due to arrhythmia related to the seizures (Tomson 2000, Cheung and Hachinski 2000, Devinsky 2004).

It is possible that the observed VNS effect on seizure and cardiac activity may somehow be exceptional. Although there is no direct evidence for the assumption, present results showing that while VNS application ameliorates PP interval prolongation and prevent BP increment, it does not totally block PTZinduced seizure but turned it into minimal clonic seizures. This may support our view.

Additionally some clinical reports also provide new evidence about the protective effect of VNS on heart rate variability and cardiac regulation in patient with epilepsy (Stember et al. 2008). These results suggested that VNS might have a positive effect on decreasing the incidence of SUDEP in patients treated with VNS (Koenig et al. 2007, Stember et al. 2008).

In conclusion present data support the relationship between seizure severity and hemodynamic abnormalities induced by epileptic seizures. VNS may have protective effects against seizure-induced changes in blood pressure and cardiac rhythm. These findings may have important implications for the management of epilepsy with VNS, because it implies that controlled seizures have a lower risk for cardiac arrhythmia.

\section{Conflict of Interest}

There is no conflict of interest.

\section{References}

BOON P, VONCK K, DE REUCK J, CAEMAERT J: Vagus nerve stimulation for refractory epilepsy. Seizure 10: 456$458,2001$.

CHEUNG RT, HACHINSKI V: The insula and cerebrogenic sudden death. Arch Neurol 57: 1685-1688, 2000.

DARBIN O, CASEBEER DJ, NARITOKU DK: Cardiac dysrhythmia associated with the immediate postictal state after maximal electroshock in freely moving rat. Epilepsia 43: 336-341, 2002.

DARBIN O, CASEBEER D, NARITOKU DK: Effects of seizure severity and seizure repetition on postictal cardiac arrhythmia following maximal electroshock. Exp Neurol 181: 327-331, 2003. 
DE HERDT V, BOON P, CEULEMANS B, HAUMAN H, LAGAE L, LEGROS B, SADZOT B, VAN BOGAERT P, VAN RIJCKEVORSEL K, VERHELST H, VONCK K: Vagus nerve stimulation for refractory epilepsy: A Belgian multicenter study. Eur J Paediatr Neurol 11: 261-269, 2007.

DEVINSKY O: Effects of seizures on autonomic and cardiovascular function. Epilepsy Curr 4: 43-46, 2004.

DOOLEY M, PLOSKER GL: Levetiracetam. A review of its adjunctive use in the management of partial onset seizures. Drugs 60: 871-893, 2000.

ILBAY G, SAHIN D, ATES N: Changes in blood-brain barrier permeability during hot water-induced seizures in rats. Neurol Sci 24: 232-235, 2003.

KOENIG SA, LONGIN E, BELL N, REINHARD J, GERSTNER T: Vagus nerve stimulation improves severely impaired heart rate variability in a patient with Lennox-Gastaut-Syndrome. Seizure 17: 469-472, 2007.

KOSTOV H, LARSSON PG, ROSTE GK: Is vagus nerve stimulation a treatment option for patients with drug-resistant idiopathic generalized epilepsy? Acta Neurol Scand Suppl 187: 55-58, 2007.

KRAHL SE, SENANAYAKE SS, HANDFORTH A: Destruction of peripheral C-fibers does not alter subsequent vagus nevre stimulation-induced seizure suppression in rats. Epilepsia 42: 586-589, 2001.

KRAHL SE, SENANAYAKE SS, HANDFORTH A: Right-sided vagus nerve stimulation reduces generalized seizure severity in rats as effectively as left-sided. Epilepsy Res 56: 1-4, 2003.

NARITOKU DK, TERRY WJ, HELFERT RH: Regional induction of fos immunoreactivity in the brain by anticonvulsant stimulation of the vagus nerve. Epilepsy Res 22: 53-62, 1995.

PAXINOS G, WATSON C: The Rat Brain in Stereotaxic Coordinates. Academic Press, San Diego, 1998.

SAHIN D, ILBAY G, ATES N: Changes in the blood-brain barrier permeability and in the brain tissue traceelement concentrations after single and repeated pentylenetetrazole-induced seizures in rats. Pharmacol Res 48: 69-73, 2003.

SAKAMOTO K, SAITO T, ORMAN R, KOIZUMI K, LAZAR J, SALCICCIOLI L, STEWART M: Autonomic consequences of kainic acid-induced limbic cortical seizures in rats: Peripheral autonomic nerve activity, acute cardiovascular changes, and death. Epilepsia 49: 982-996, 2008.

SALINSKY MC, UTHMAN BM, RISTANOVIC RK, WERNICKE JF, TARVER WB: Vagus nerve stimulation for the treatment of medically intractable seizures. Results of a 1-year open-extension trial. Vagus Nerve Stimulation Study Group. Arch Neurol 3: 1176-1180, 1996.

SCHRAEDER PL, LATHERS CM: Paroxysmal autonomic dysfunction, epileptogenic activity and sudden death. Epilepsy Res 3: 55-62, 1989.

STEMPER B, DEVINSKY O, HAENDL T, WELSCH G, HILZ MJ: Effects of vagus nerve stimulation on cardiovascular regulation in patients with epilepsy. Acta Neurol Scand 117: 231-236, 2008.

TOMSON T: Mortality in epilepsy. J Neurol 247: 15-21, 2000.

VELÍŠEK L: Models of chemically-induced acute seizures. In: Models of Seizures and Epilepsy, PITKÄNEN A, SCHWARTZKROIN PA, MOSHÉ SL (eds), Elsevier, Amsterdam, 2006, pp 127-152.

VELÍŠEK L, KUBOVÁ H, POHL M, STAŇKOVÁ L, MAREŠ P, SCHICKEROVÁ R: Pentylenetetrazol-induced seizures in rats: an ontogenetic study. Naunyn-Schmiedebergs Arch Pharmacol 346: 588-591, 1992.

VONCK K, VAN LAERE K, DEDEURWAERDERE S, CAEMAERT J, DE REUCK J, BOON P: The mechanism of action of vagus nerve stimulation for refractory epilepsy: the current status. J Clin Neurophysiol 18: 394-401, 2001.

WANNAMAKER BB: Autonomic nervous system and epilepsy. Epilepsia 26: 31-39, 1985.

WASTERLAIN CG: Mortality and morbidity from serial seizures. An experimental study. Epilepsia 15: 155-176, 1974.

WHELESS JW, MAGGIO V: Vagus nerve stimulation therapy in patients younger than 18 years. Neurology 24: 21-25, 2002.

ZABARA J: Inhibition of experimental seizures in canines by repetitive vagal stimulation. Epilepsia 33: 1005-1012, 1992.

ZIJLMANS M, FLANAGAN D, GOTMAN J: Heart rate changes and ECG abnormalities during epileptic seizures: prevalence and definition of an objective clinical sign. Epilepsia 43: 847-854, 2002. 